\title{
Experimental and Numerical Investigation of Flows in Expanding Channels
}

\author{
Peter Vorobieff and Vakhtang Putkaradze \\ kalmoth@unm.edu, vakhtang@unm.edu \\ Department of Mechanical Engineeering \\ The University of New Mexico, MSC01 115, Albuquerque, NM 87131
}

\section{Program Scope}

In any machinery operating with fluids, expanding or contracting ducts are ubiquitous. In the simplest idealized case, a duct has planar walls, expanding or contracting in the $x-y$ plane, with the $x-y$ cross-section $z$-independent for some considerable range of $z$. For a slow-moving fluid flow in this geometry, a simple solution exists which can be described by analytical formulæ (Jeffery-Hamel, or $\mathrm{JH}$, flow) $)^{1,2}$. $\mathrm{JH}$ solutions are perhaps the most fundamental in fluid mechanics; they concern two-dimensional stationary flows of an incompressible viscous fluid in an idealized expanding (contracting) channel, or wedge. Moreover, modifications and generalizations of the $\mathrm{JH}$ similarity solution have provided analytical templates for a large variety of exact solutions of the 2D boundary layer equations for corner, wedge, sink, and other flows. In JH flows, the characteristic flow rate is usually described in terms of the Reynolds number $R=Q / v$, where $Q$ is volume flux per unit length of the source in the $z$-direction and $v$ is the fluid kinematic viscosity. For a symmetric channel, the flow for small $R$ is symmetric, with maximum velocity in the center. When the Reynolds number increases, this simple solution ceases to exist. The flow breaks the symmetry (bifurcates), with most of the fluid going in a thin layer along one wall. The fluid is prevented from utilizing the whole area of the expanding channel by a recirculation vortex which blocks the exit. In addition, secondary instabilities driven by this vortical motion develop in this flow. This proposal addresses the least studied regime of the intermediate Reynolds numbers separating the zones of low- $R$ nearly-2D deterministic flow and high- $R$ turbulent flow which can be assessed statistically. We study both the stationary bifurcations and the non-stationary transitional flow features. The understanding of the flow physics is achieved through a combination of experimental, theoretical and numerical studies. This study is also of interest as an experimental benchmark for numerical code validation.

\section{Progress Summary}

The subtly intricate nature of the JH solutions and their practical importance motivated many stability studies of JH flows. A serious problem for the stability analysis is the lack of realistic boundary conditions at the outlet, while the choice of different boundary conditions can lead to different results. This would tremendously complicate any purely theoretical investigation of the selection mechanism and solution stability in JH flow. However, these ambiguities related to the boundary conditions can be trivially resolved in experiment. Quite surprisingly, until the results of our work sponsored by the DOE EPSCoR grant were published, there had been no consistent experimental study of JH flows and their bifurcations.

Figure 1 presents a summary of the possible radial velocity profiles in wedge flows. The simplest are the pure inflow ("I") and pure outflow ("O") solutions, corresponding to a sink or a source at the narrowest contraction point of the channel. An asymmetric profile (IO) is also 
known to be realized. Another symmetric profile with two inflow zones (IOI) is known from theory.

In our experiment, JH flows are realized in a long $(38.6 \mathrm{~cm}$ in the direction parallel to the visualization axis) rectangular acrylic container placed inside a much larger water tank (total volume $60 \mathrm{l}$ ). The container has a $0.2 \mathrm{~cm}$ wide slit in the bottom. Two $10.2 \mathrm{~cm}$ long inclined walls are attached at a fixed angle to the bottom near the slit. Multiple containers were fabricated, with the angle between the walls varying from 12 to 60 degrees. The flow field is visualized by illuminating a planar section of the flow (corresponding to the $x-y$ plane), with a pulsed laser sheet. The fluid in the container (water) is seeded with a small volume fraction of tracer particles (titanium dioxide flakes). A high-resolution digital camera captures the images of the flow fields both for visualization purposes and for quantitative acquisition of the flow fields using particle image velocimetry (PIV) ${ }^{3}$.

Our results confirm the stability of the pure outflow (Fig. 1, "O") solution, whenever it exists. As the flow rate in the outflow regime and the Reynolds number increase, a different solution is realized, with the radial velocity profile roughly corresponding to the asymmetrical solution (Fig. 1, "IO"). In reality, the velocity vectors in this flow are no longer oriented in the radial direction (as was the case for the low- $R$ pure outflow). Instead, a large recirculating vortex forms and attaches itself to one of the walls. However, the large-scale structure of the flow remains spatially two-dimensional.
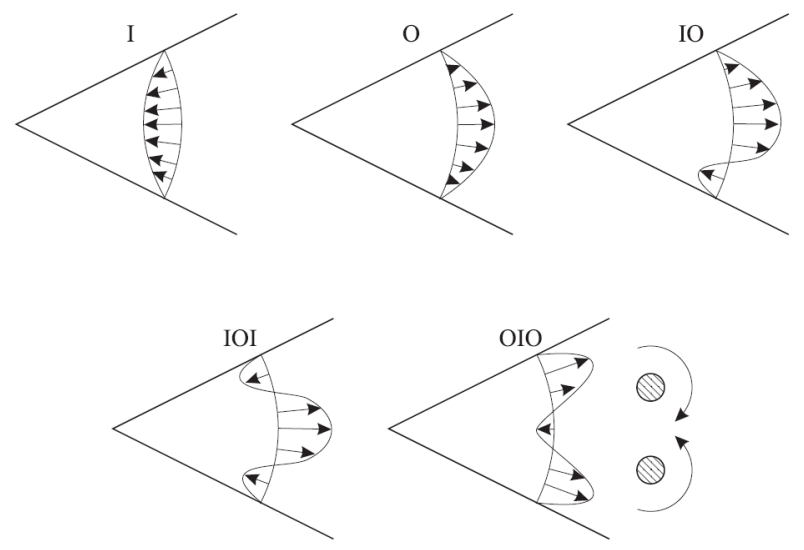

Figure 1. Flow regimes in different types of Jeffery-Hamel flows. Each type of flow is labeled by a different type of symbols I (inflow) and $\mathrm{O}$ (outflow).

We found that the value of the Reynolds number characterizing the transition between the symmetric radial outflow regime and the vortex-dominated asymmetric regime depends on the direction of the transition. In other words, as we increase the flow rate, the critical Reynolds number at which the flow transitions to the asymmetric regime is higher than the corresponding Reynolds number for the reverse transition as the flow rate is decreased - the phenomenon known as hysteresis. The existence of hysteretic transition between these flow regimes was quite surprising, as it has no explanation within the traditional $\mathrm{JH}$ theory.

Instead, we attribute the existence of this hysteresis to a temporally transient, convective (in the sense that it propagates downstream with the flow) instability. If this instability develops slowly enough, it is flushed out of the system without changing the flow pattern. However, if vortex roll-up due to this instability occurs on the same or smaller time scale as the time it takes for the fluid to exit the system, the flow is destabilized. The delay between the transition to vortex-dominated flow as the Reynolds number is increased is successfully explained by 
invoking this destabilization mechanism.

Notably, secondary shear-driven instabilities of the large vortex manifested themselves as soon as the vortex formed. This observation was consistent with the results of separate sheardriven instability studies carried out in a novel quasi-two-dimensional shear flow generator based on a soap film tunnel ${ }^{4}$. In addition, that investigation revealed some features peculiar to soap film systems. Interaction with air surrounding the quasi-two-dimensional flow in the film of soap suspended between two nylon wires led to preferential removal of energy from vortices of large scales, and since in two-dimensional turbulence, unlike its better known three-dimensional analog, the energy cascade moves kinetic energy from smaller vortices to larger ones, this energy removal led to formation of fossil turbulence structures advected by the flow but no longer evolving.

Recently, an interesting stationary solution in JH flow geometry has been discovered 5 . The solution consists of periodically spaced vortices attached alternatively to either left or right walls. The boundary conditions for this solution have to be periodic in the inlet and outlet. The apparently nonphysical boundary conditions notwithstanding, we have managed to realize such a solution in our experiment (Fig. 2). This solution is transient, but the time scale over which it decays ( 10 minutes) is much longer than the equilibration time for this regime. The decay of this solution happens when the inner vortex "squeezes out" the outer vortex and thus is due to the discrepancy in boundary conditions.
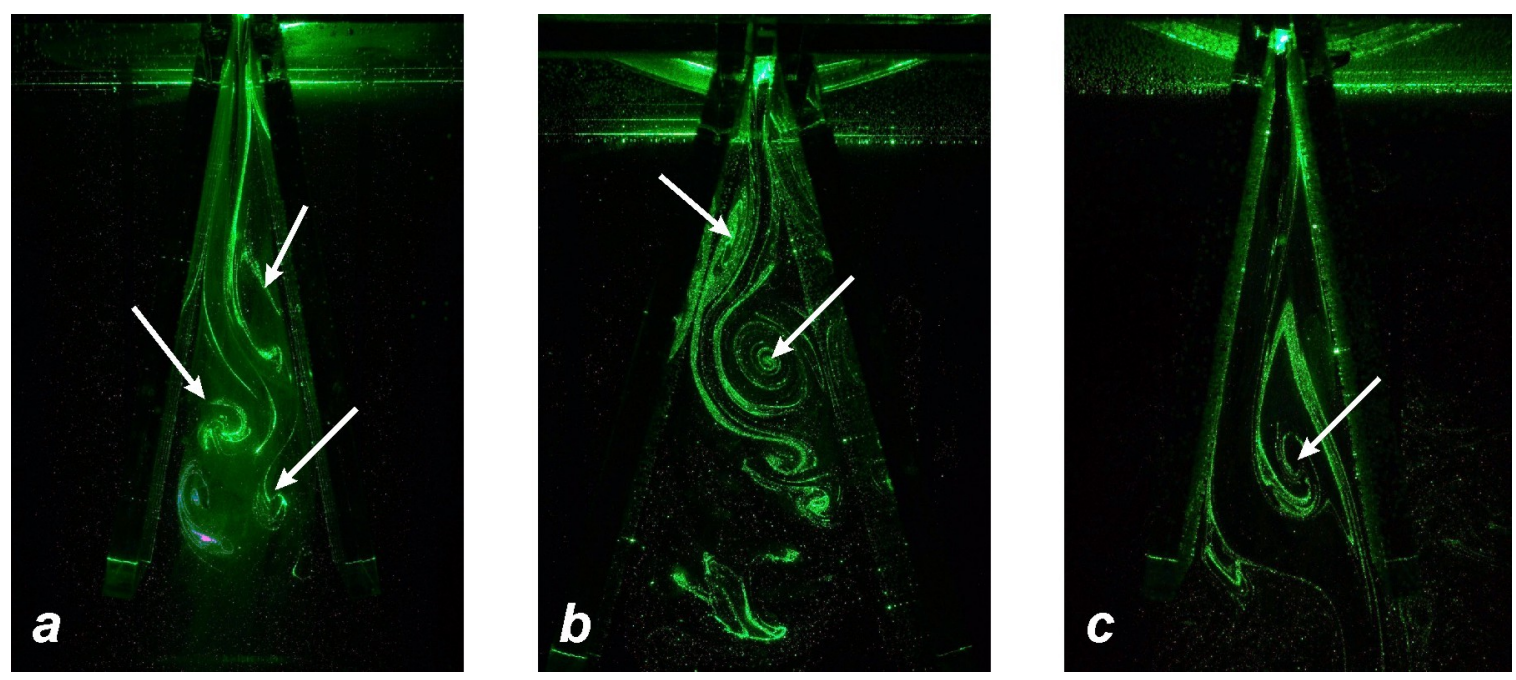

Figure 2. In some post-transient regimes, a pattern of long-lived alternating vortices forms. A notion of a spatial periodicity of this pattern could be inferred from the fact in the $21^{\circ}$ angle wedge (a) three such vortices are present, whereas in the $32^{\circ}$ wedge (b) we observe only two of them. This pattern might match the stationary Tutty solution, obtained for a wedge flow with periodic boundary conditions. However, as the boundary conditions in experiment are not the same, the innermost vortex "squeezes out" the outer one(s) and attaches to one of the walls, leading to a stationary asymmetrical solution (c).

We have also investigated the influence of boundary conditions on the selection of the morphology that is realized in the flow. The boundary conditions are known to influence the stability of the solutions. We used several combinations of rotors/impellers in combination with a $32^{\circ}$ wedge, as shown in Fig. 3, in an attempt to destabilize the flow or to realize flow regimes 
that do not occur naturally in wedge flows. Here we refer to the impeller action as "blowing" if the impeller drives water into the wedge, and "suction" if it drives it into the opposite direction. Blowing and suction can be symmetric (with both impellers counter-rotating at same angular velocity) or asymmetric, with only one impeller rotating. With both symmetric and asymmetric suction, we found it very difficult to destabilize the flow in the regimes where the radial outflow solution is nominally stable. Namely, the characteristic flow rate from the impeller had to be on the same order as the discharge rate from the slit. Mild actuation did not change the character of the flow structure at $R \sim 25$. Interestingly enough, mild symmetric and asymmetric suction (increased proportionally to Reynolds number) did not have much effect on the vortexdominated flow at $R \sim 45$ either.

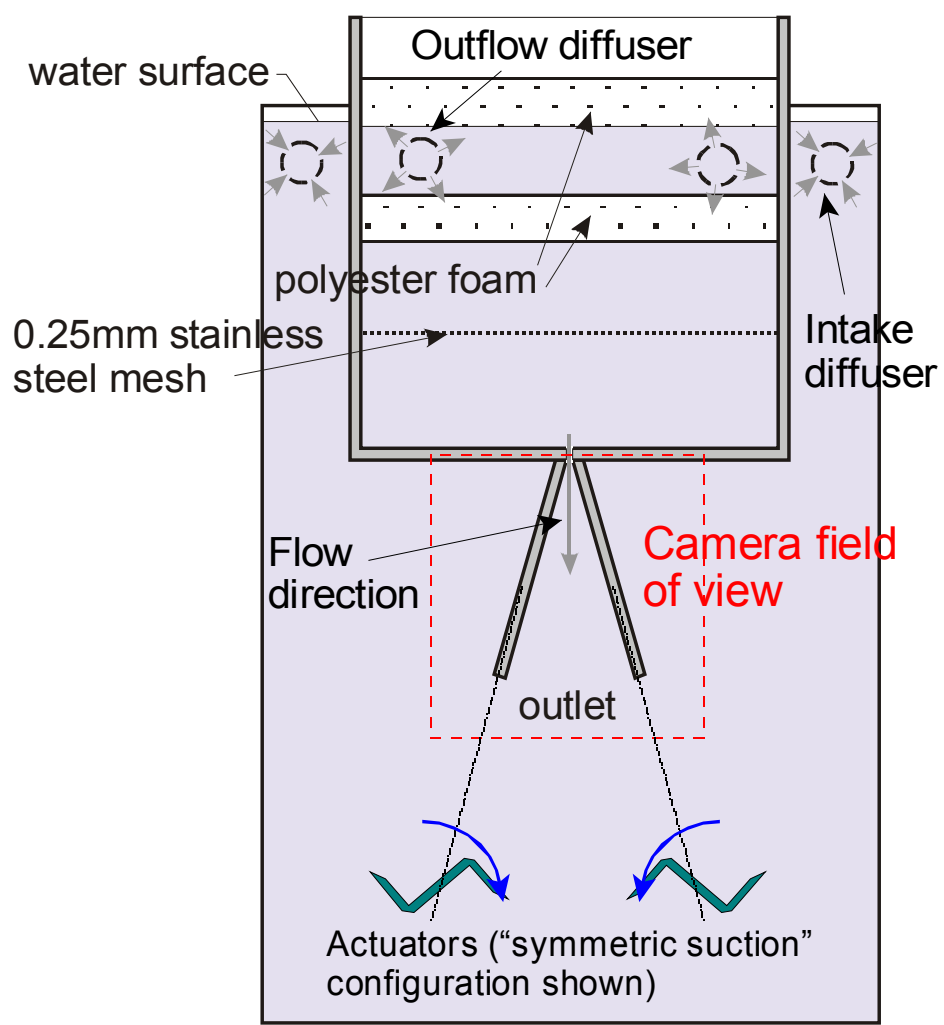

Figure 3. Schematic of the experimental arrangement with actuators (impellers) affecting the wedge flow.

However, we found that mild symmetric suction appears to raise the threshold of the transition from asymmetric flow to symmetric flow as $R$ is decreased from 45 to 25 (thus effectively stabilizing the flow in the bifurcation region). Similarly, mild asymmetric blowing accelerates transition from symmetric to vortex-driven flow in the bifurcation region.

\section{Future work and collaborations}

Our results indicate that the transition between symmetric and asymmetric flow regimes is affected by actuation shown in Fig. 3, however, a more detailed study of the phase space of the system (including configurations with multiple alternating impellers, etc). still needs to be undertaken. Moreover, compressibility effects are likely to play a role in transition between 
symmetric and asymmetric flow regimes. We have consulted with Dr. Timothy Madden (AFRL) about the possibility of conducting a joint experimental/numerical work including a study of these effects. Our proposal to AFOSR to fund this investigation is currently under review.

\section{References}

(1) Jeffery, G.B., "The Two-Dimensional Steady Motion of a Viscous Fluid," Phil. Mag. Vol. 29 (6), pp. 455-465 (1915).

(2) Hamel, G., "Spiralförmige Bewegungen zäher Flüssigkeiten," Jahresbericht Deutsch. Math. Verein Vol. 25, pp. 34-60 (1916).

(3) Grant, I., "Particle image velocimetry: A review," Proc. Inst. Mech. Eng., Part C: J. Mech. Eng. Sci. Vol. 211 no.1, pp. 55-76 (1997).

(4) Georgiev, D., and Vorobieff, P., "The slowest soap-film tunnel in the Southwest," Rev. Sci. Instrum. Vol. 73 (3), pp. 1177-1184 (2002).

(5) Kerswell, R.R., Tutty, O.R., and Drazin, P.G., "Steady nonlinear waves in diverging channel flow," J. Fluid Mech. Vol. 501, pp. 231-250 (2004).

\section{Supported Graduate Students}

Aparna Korlimarla, M.S. (2006)

Tanveer Shakeel, Ph.D. (2007)

Keith Mertens, Ph. D. (2008)

\section{DOE Sponsored Publications}

Holm, D.D., Putkaradze, V., Weidman, P. D., and Wingate, B., "Boundary effects on exact solutions of the Lagrangian-averaged Navier-Stokes equations," Journal of Statistical Physics vol. 113 (5/6), pp. 841-854 (2003).

Holm, D.D., Putkaradze, V., and Stechmann, S., "Rotating Cocentric Circular Peakons," Nonlinearity vol. 17, pp. 1-24 (2004).

Putkaradze, V., Durgin, T., Vorobieff, P., and Deshler, J., "Hysteresis in Jeffery-Hamel flows: experiments and theory", American Physical Society, 57th Annual Meeting of the Division of Fluid Dynamics, November 21-23, 2004, abstract \#GP.002.

Korlimarla, A., and Vorobieff, P., "Evolution of a quasi-2D shear layer in a soap film flow," American Physical Society, 57th Annual Meeting of the Division of Fluid Dynamics, November 21-23, 2004,abstract \#GG.008.

Putkaradze, V. and Vorobieff, P, "Instabilities, Bifurcations, and Multiple Solutions in Expanding Channel Flows,” Physical Review Letters vol. 97, art. no. 144502 (2006).

Vorobieff, P., and Putkaradze, V., "Jeffery-Hamel flow: an experimental study of instabilities, bifurcations and multiple solutions," American Physical Society, 59th Annual Meeting of the APS Division of Fluid Dynamics, November 19-21, 2006, abstract \# AO.009. 
Nitsche, M., Holm, D.D., and Putkaradze, V., "Euler-alpha and vortex blob regularization of vortex filament and vortex sheet motion," Journal of Fluid Mechanics vol. 555, pp. 149-176 (2006).

Korlimarla, A., "Turbulence in two-dimensional shear flows," M.Sc. Thesis, The University of New Mexico (2006).

Putkaradze, V., and Watanabe, S., "A simple model for description of flows in symmetric channel expansions," Physics Letters A, vol 370, pp. 58-63 (2007).

Vorobieff, P., and Putkaradze, V. "Flow regimes inside an expanding channel," American Physical Society, $60^{\text {th }}$ Annual Meeting of the APS Division of Fluid Dynamics, November 1820, 2007, abstract no. AL.005.

Putkaradze, V., and Watanabe, S., "Extended boundary layer theory and modeling of flows in symmetric channel expansions," Fluid Dynamics Research, under consideration (2008).

Vorobieff, P., and Putkaradze, V., "Expanding channel flows: stability, bifurcations and boundary conditions," in preparation (2008). 